Phospholipase $\mathrm{A}_{2}$ Inhibitor-Loaded Phospholipid Micelles Abolish

\title{
Neuropathic Pain
}

Sonia Kartha, ${ }^{1}$ Lesan Yan, ${ }^{1}$ Meagan E. Ita, ${ }^{1}$ Ahmad Amirshaghaghi, ${ }^{1}$ Lijun Luo, ${ }^{1}$ Yulong Wei, ${ }^{1}$ Andrew Tsourkas, ${ }^{1}$ Beth A. Winkelstein,1,2 and Zhiliang Cheng ${ }^{1}$.

${ }^{1}$ Department of Bioengineering, University of Pennsylvania, 210 South 33rd Street, 240 Skirkanich Hall, Philadelphia, PA 19104, USA

${ }^{2}$ Department of Neurosurgery, University of Pennsylvania, Hospital of the University of Pennsylvania, 3400 Spruce Street, 3 Silverstein, Philadelphia, PA 19104 


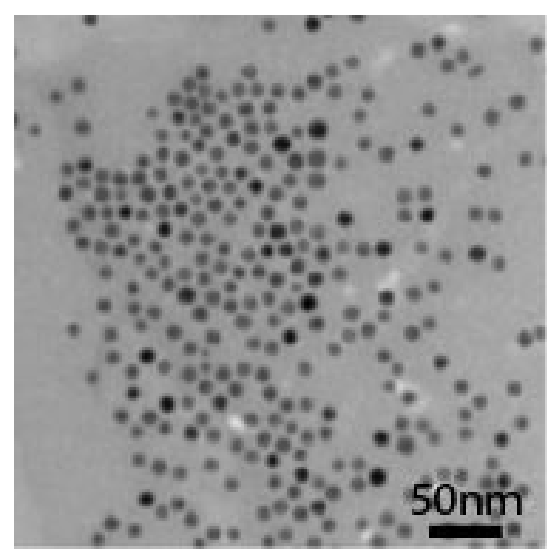

Figure s1. Transmission electron microscopy (TEM) image of individual hydrophobic SPION. 


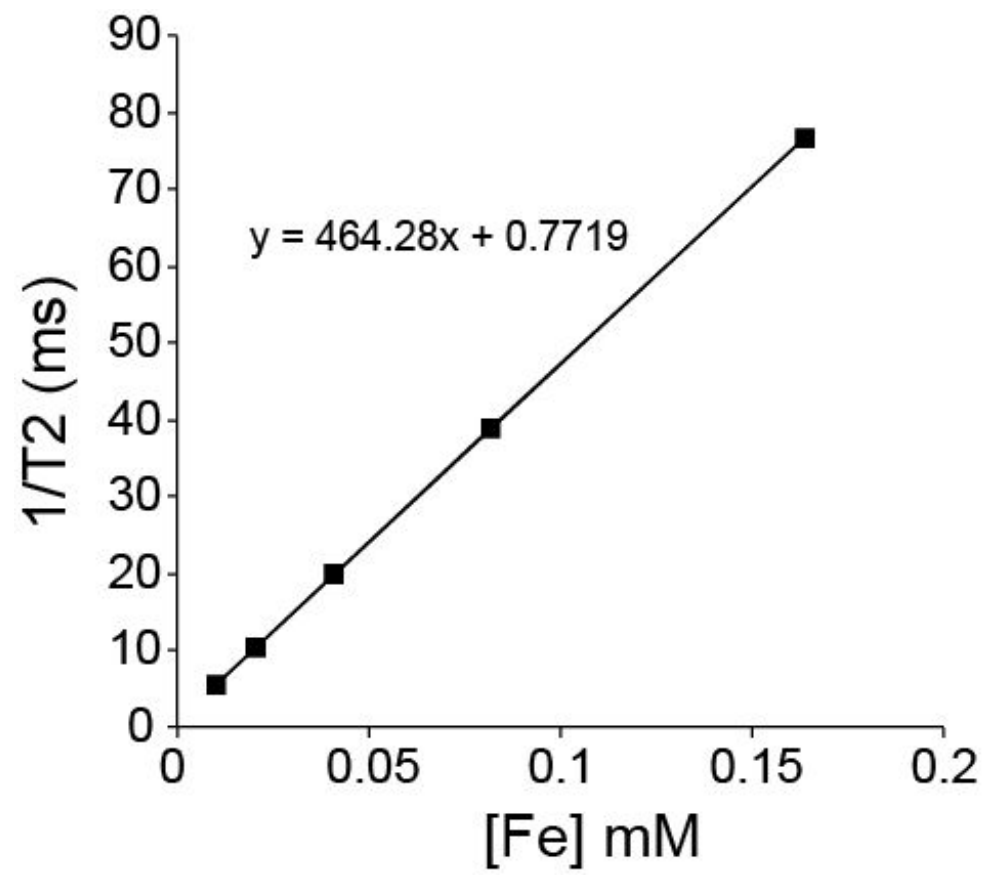

Figure s2. Relaxivity determination for SPLA $_{2}$ inhibitor-loaded micelles. 


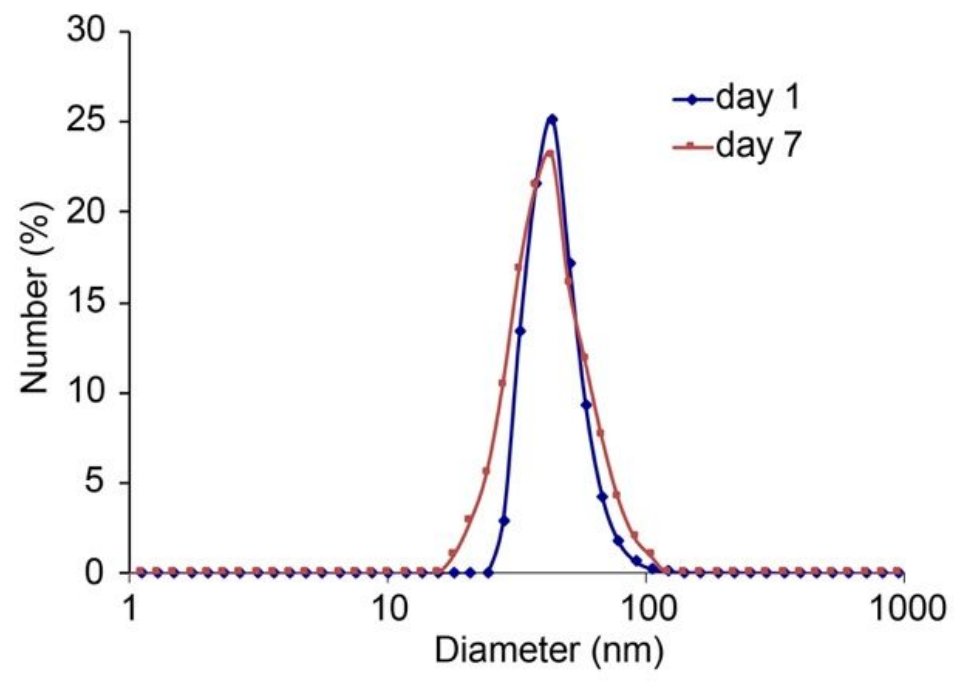

Figure s3. Incubation of $\mathrm{SPLA}_{2}$ inhibitor-loaded micelles in buffer (0.1 M PBS, pH 7.4) does not change micelle stability for at least a week with no observable change in the hydrodynamic diameter of the micelle during incubation. 


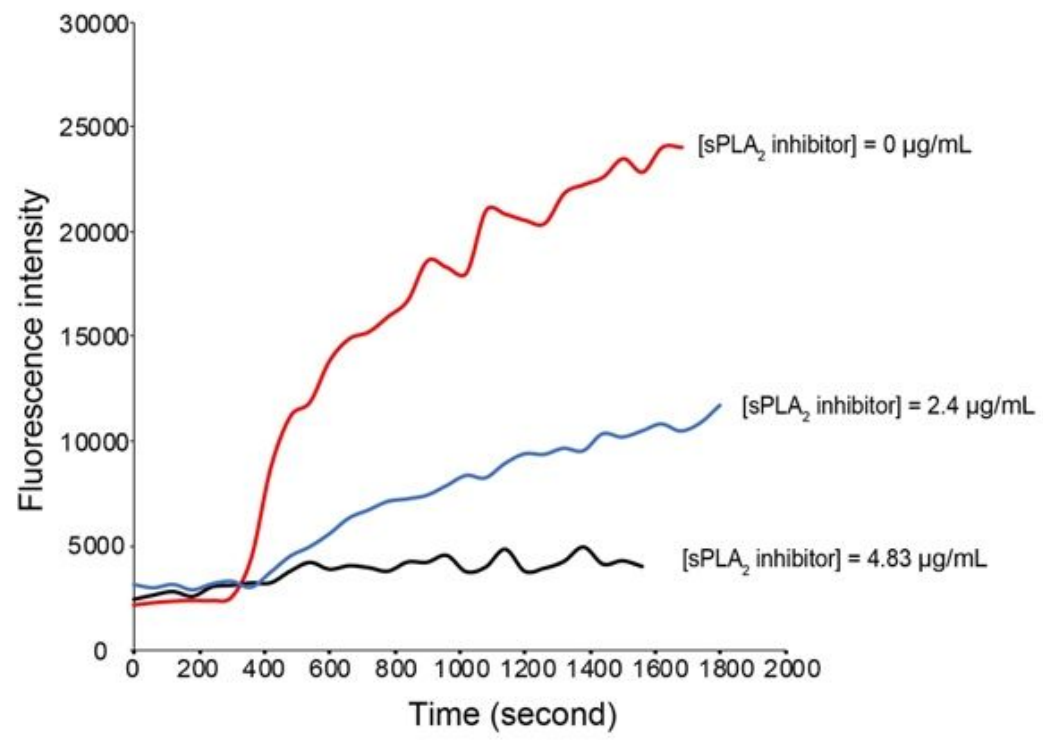

Figure s4. sPLA 2 inhibitor-loaded micelles inhibited sPLA 2 activity in a concentration dependent manner as observed by a reduction in fluorescence activation. 


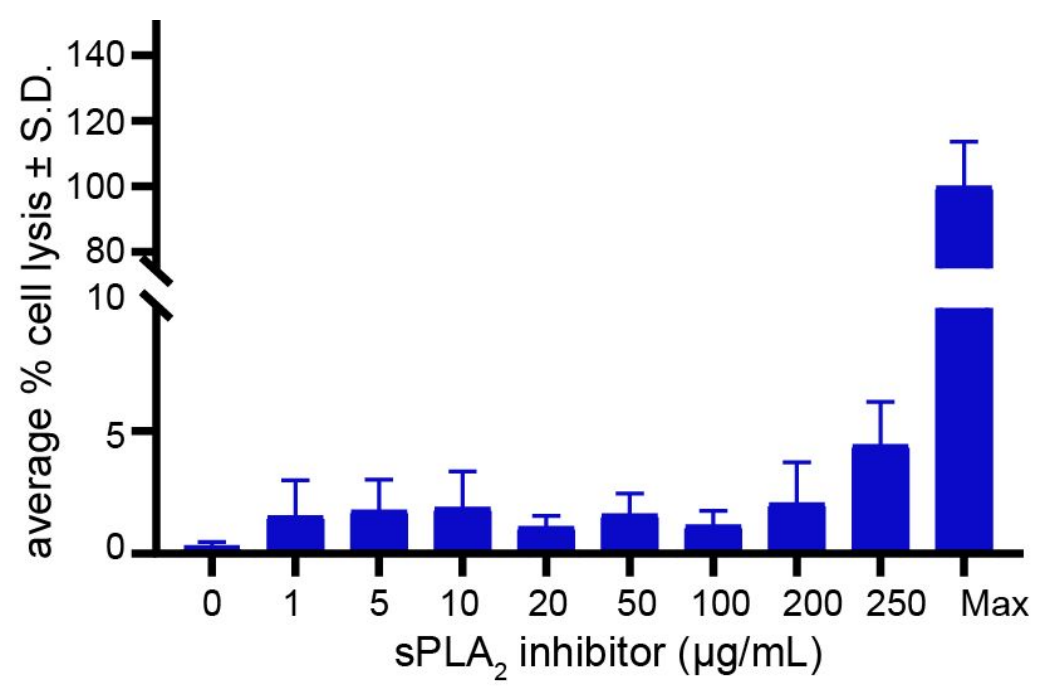

Figure s5. Incubation with SPLA $_{2}$ inhibitor-loaded micelles does not significantly increase the average percent of cell lysis observed in primary DRG cultures, with no significant differences $(p>0.38)$ for any micelle concentrations compared to control. 


\section{contralateral nerve root}

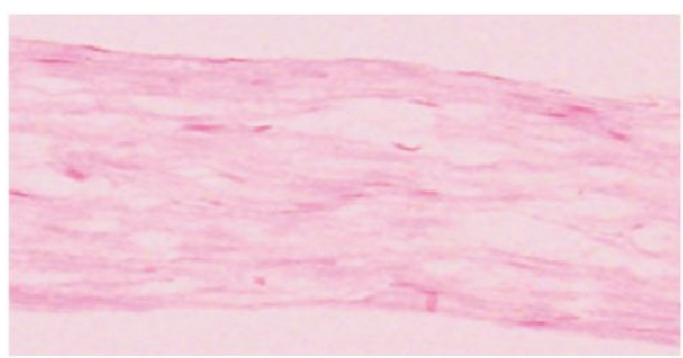

ipsilateral nerve root

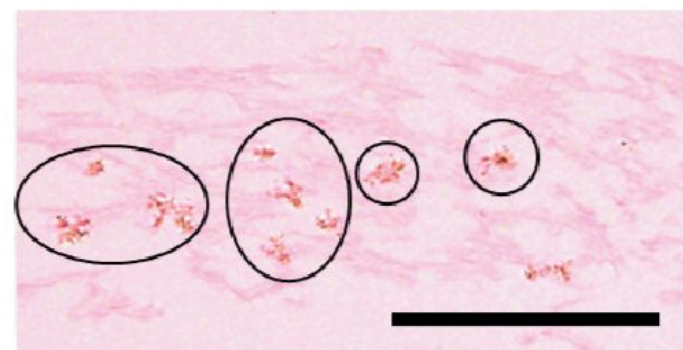

Figure s6. Unloaded micelles (i.e. without sPLA $_{2}$ inhibitor) are found to localize at day 7 to the injured nerve root after local administration at the time of injury (day 0). Iron is detected (circles) only in the ipsilateral C7 dorsal nerve root after a nerve root compression that is treated with unloaded micelles. There is no evidence of iron in the contralateral C7 nerve root after either type of treatment. The scale bar is $100 \mu \mathrm{m}$ and applies to all panels. 
A

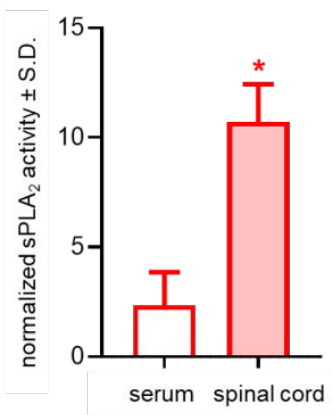

B

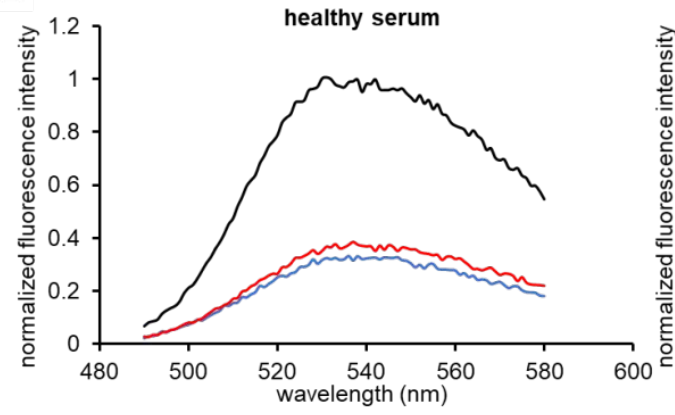

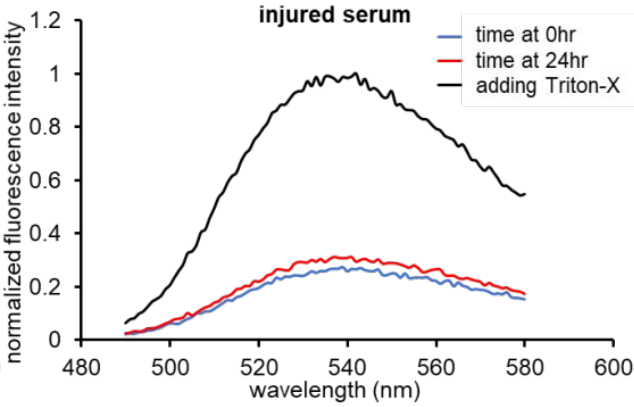

Figure s7. (A) sPLA $_{2}$ activity assay of serum and spinal cord tissue collected one day after a painful injury shows significantly increased $(p=0.0004)$ activity in the spinal cord compared to serum. (B) Incubation $\mathrm{sPLA}_{2}$-responsive phospholipid liposomes with serum from injured rats does not affect the fluorescence release from $\mathrm{SPLA}_{2}$ responsive phospholipid liposomes at 0 or 24 hours compared to incubation with healthy serum and is lower than Triton$\mathrm{X}$ positive controls. 


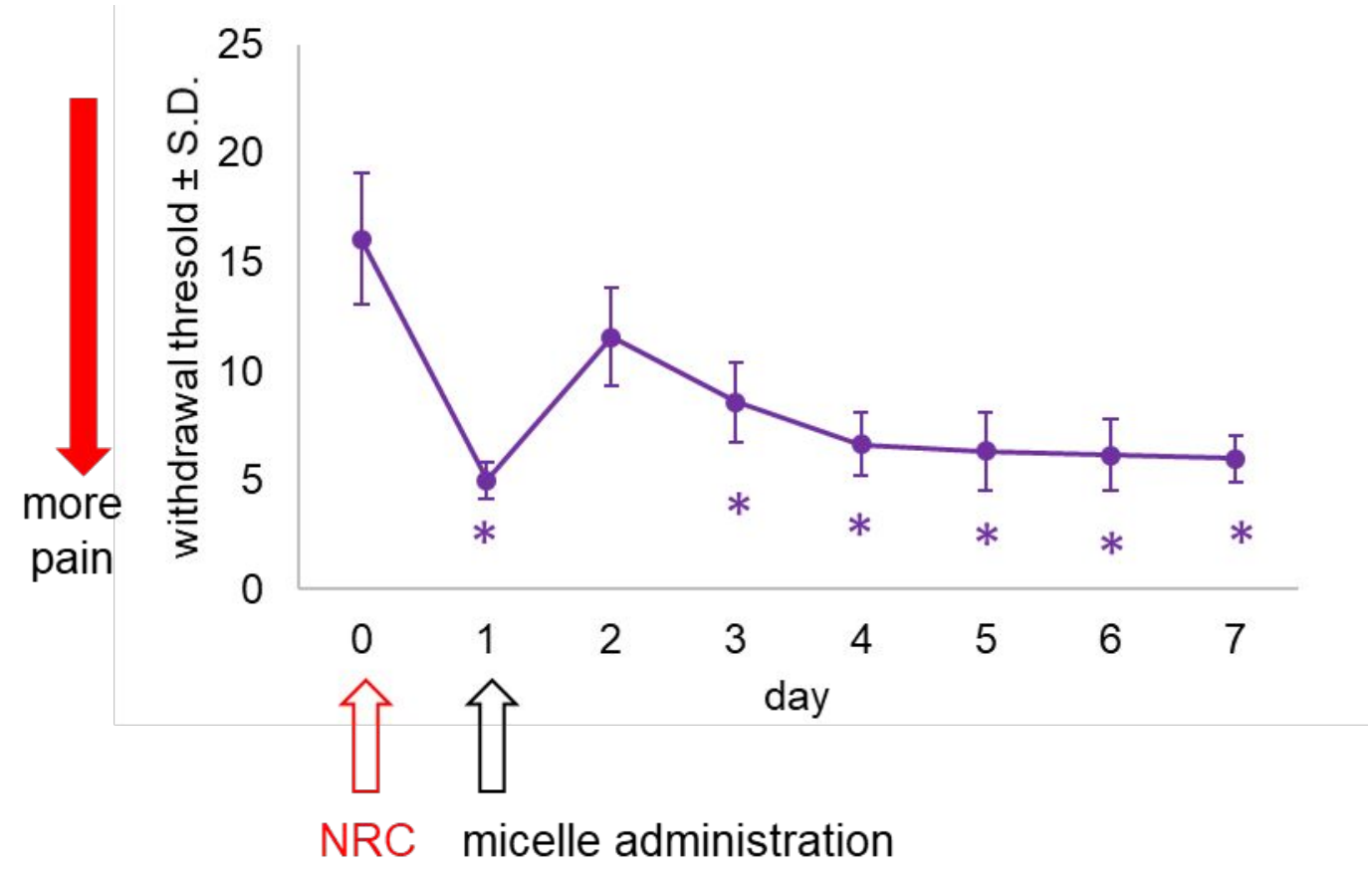

Figure s8. Following painful nerve root compression on day 0 , withdrawal thresholds on day 1 are significantly lower $(p<0.0002)$ than pre-injury (day 0) levels. Administration of a single dose of sPLA $_{2}$ inhibitor-loaded micelles on day 1 transiently increases thresholds on day 2, but thresholds are significantly lower ( $p<$ 0.0007 ) on days 3 through 7 indicating the presence of pain on those day. 


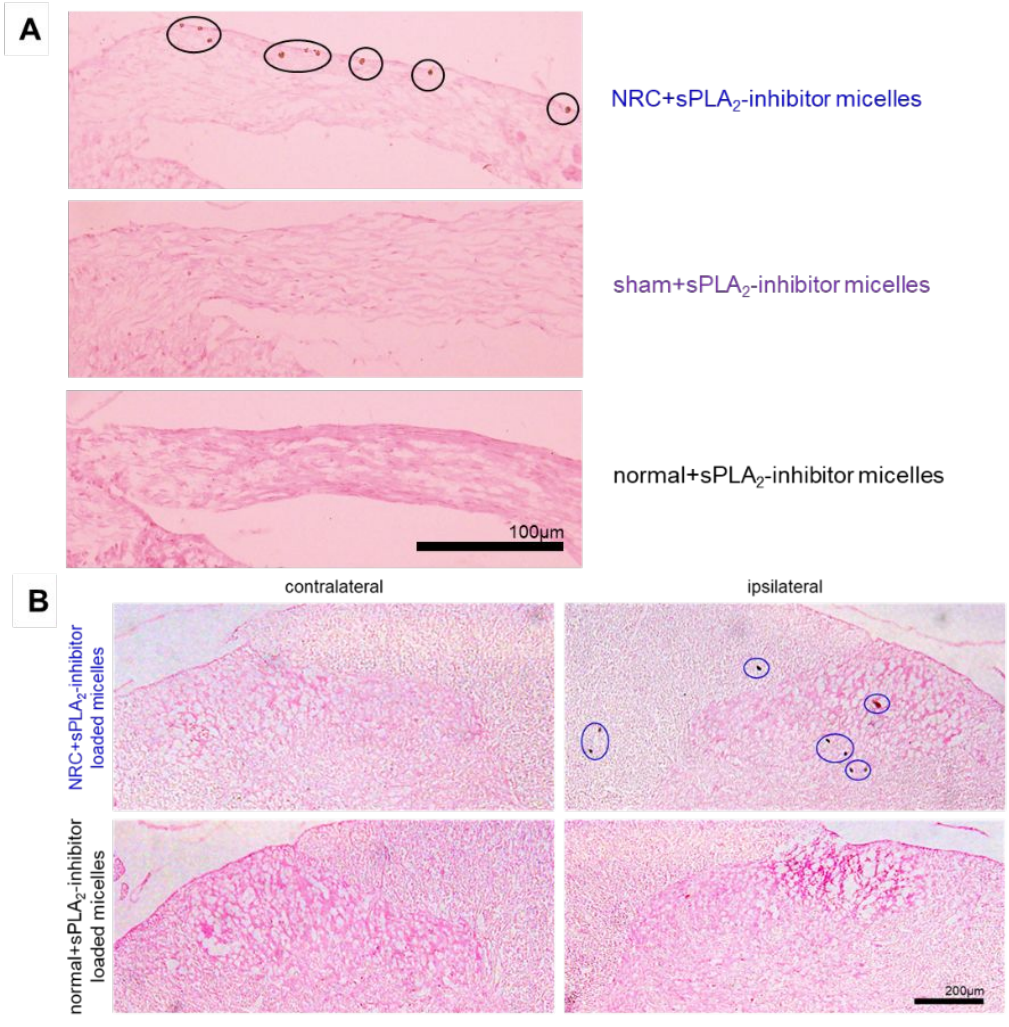

Figure s9. (A) At day 7 after intravenous administration of $\mathrm{sPLA}_{2}$ inhibitor-loaded micelles, Prussian Blue- labeled SPIOs only localize to the injured nerve root and not to uncompressed sham and normal nerve roots. (B) Positive SPIO labeling is also observed in the white and gray matter only in the ipsilateral spinal cord following intravenous administration of $\mathrm{SPLA}_{2}$ inhibitor-loaded micelles. There was no SPIO labeling evident in the ipsilateral or contralateral spinal sections of naive rats treated with $\mathrm{sPLA}_{2}$ inhibitor-loaded micelles. 


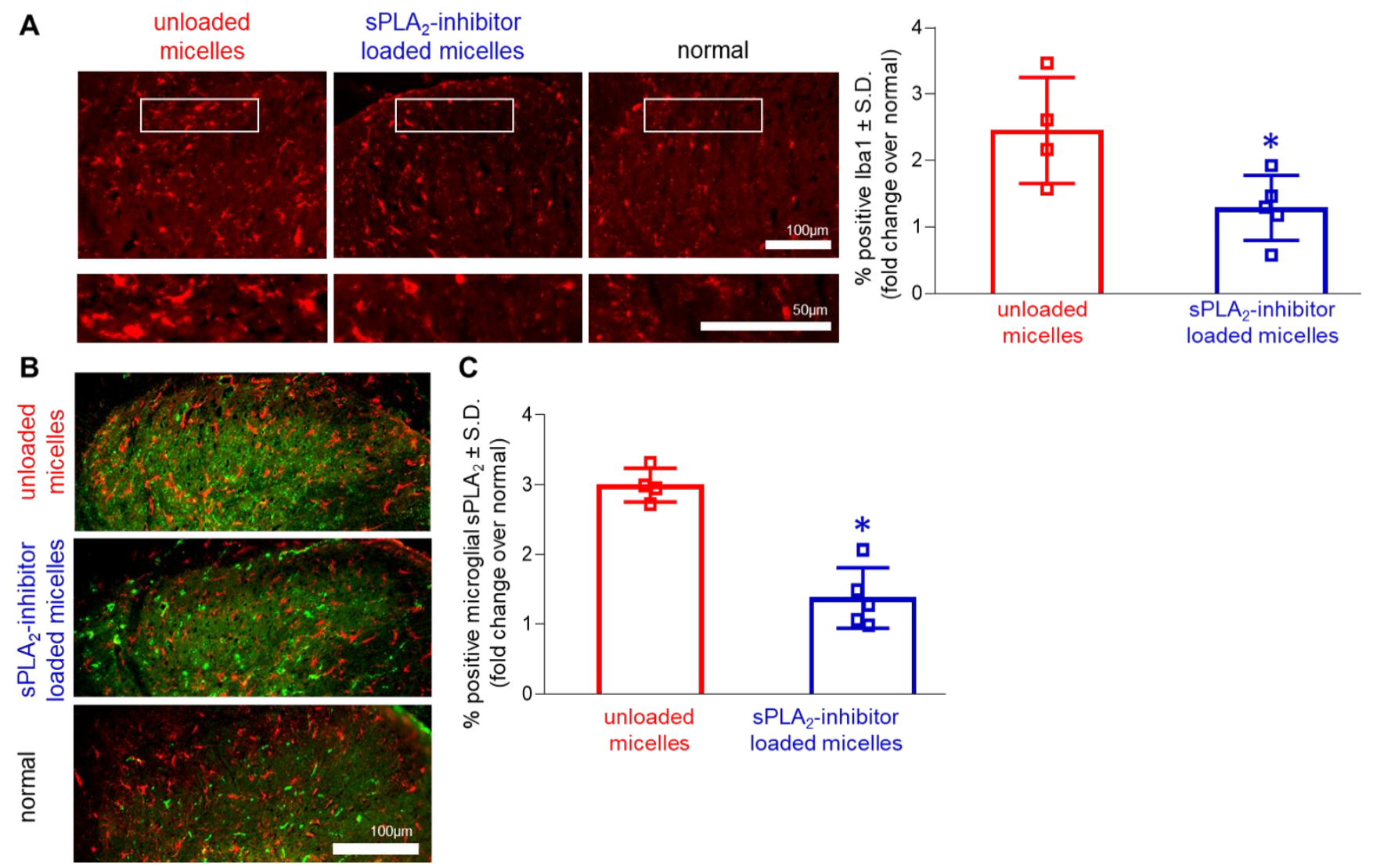

Figure s10. (A) Spinal Ibal expression at day 7 is significantly elevated $(* p<0.0002)$ following systemic treatment with unloaded micelles compared to systemic treatment with SPLA $_{2}$ inhibitor-loaded micelles. The images on the bottom row are higher magnification of the enclosed boxes to show individual cells. (B) Representative images of the spinal dorsal horn show a reduction in microglial SPLA $_{2}$ (yellow) following treatment with SPLA $_{2}$ inhibitor-loaded micelles. (C) Spinal microglial $\mathrm{sPLA}_{2}$ expression is significantly reduced $\left({ }^{*} p=0.0002\right)$, to normal levels, with sPLA ${ }_{2}$ inhibitorloaded micelles compared to treatment with unloaded micelles. 
A

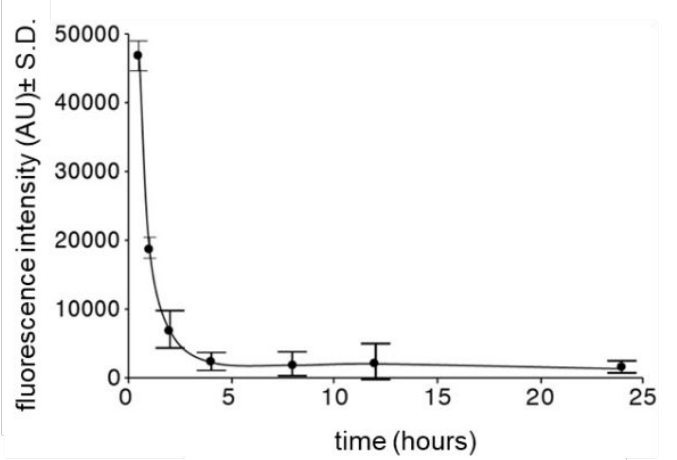

B

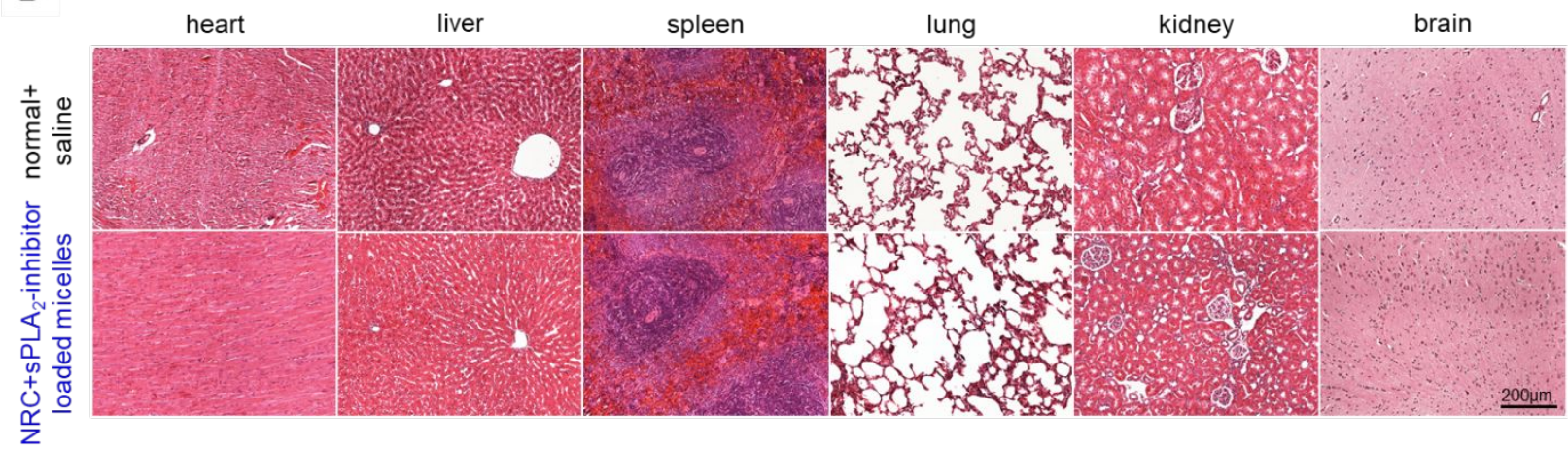

Figure s11. (A) The pharmacokinetic profile of rhodamine-labeled $\mathrm{SPLA}_{2}$ inhibitor-loaded micelles in whole blood following intravenous administration indicates that micelles have an in vivo half-life of around 45 minutes. (B) H\&E staining of rat heart, liver, spleen, lung, kidney and brain at 7 days after intravenous injection of sPLA $_{2}$ inhibitor-loaded micelles or saline show no abnormalities. 